\title{
Initial management of noncirrhotic splanchnic vein thrombosis: When is anticoagulation enough?
}

\author{
Pranavi Ravichandran MD ${ }^{1}$, Kris P Croome MD MS ${ }^{1,2}$, Michael J Kovacs MD FRCPC ${ }^{3}$, \\ Alejandro Lazo-Langner MD MSc ${ }^{3,4}$, Roberto Hernandez-Alejandro MD ${ }^{1,2}$
}

P Ravichandran, KP Croome, MJ Kovacs, A Lazo-Langner, $\mathrm{R}$ Hernandez-Alejandro. Initial management of noncirrhotic splanchnic vein thrombosis: When is anticoagulation enough? Can J Gastroenterol Hepatol 2014;28(4):207-211.

BACKGROUND: The optimal initial treatment of splanchnic vein thrombosis is uncertain. Anticoagulant therapy has been shown to be associated with vessel recanalization and decreased recurrence. Furthermore, information regarding potential predictors of chronic complications is not well understood.

METHODS: A retrospective cohort study involving consecutive patients diagnosed with first-episode noncirrhotic splanchnic vein thrombosis referred to the thrombosis clinic of the authors' institution between 2008 and 2011 was conducted. Demographic and clinical information was collected. The response to initial anticoagulant therapy was evaluated by determining radiographic recanalization of vessels and clinical resolution (defined as the absence of ongoing splanchnic vein thrombosis symptoms or complications requiring treatment beyond anticoagulant therapy).

RESULTS: Twenty-two patients were included. Anticoagulant therapy alone resulted in vessel recanalization in $41 \%$ of patients and $68 \%$ achieved clinical resolution. Two patients experienced bleeding events. Factors associated with a lack of clinical resolution included signs of portal hypertension/liver failure on presentation, complete vessel occlusion at diagnosis, presence of a myeloproliferative disorder or JAK2 $2^{\mathrm{V} 617 \mathrm{~F}}$ tyrosine kinase mutation and the absence of a local/ transient predisposing factor.

CONCLUSIONS: Anticoagulant therapy appeared to be an effective initial treatment in patients with splanchnic vein thrombosis. Clinical factors may help to identify patients who are at risk for developing complications thus requiring closer monitoring. These findings were limited by the small sample size and need to be explored in larger prospective studies.

Key Words: Anticoagulation; Portal hypertension; Splanchnic vein thrombosis; Therapy

\author{
La prise en charge initiale de la thrombose veineuse \\ splanchnique non cirrhotique : quand \\ l'anticoagulation suffit-elle?
}

HISTORIQUE : On ne connaît pas le traitement initial optimal de la thrombose veineuse splanchnique. L'anticoagulothérapie s'associe à une recanalisation des vaisseaux et à une diminution des récurrences. De plus, on comprend mal les données sur les prédicteurs potentiels des complications chroniques.

MÉTHODOLOGIE : Les auteurs ont mené une étude rétrospective de cohorte auprès de patients consécutifs ayant un diagnostic de premier épisode de thrombose veineuse splanchnique aiguillés vers la clinique de thrombose de leur établissement entre 2008 et 2011. Ils ont colligé des renseignements démographiques et cliniques. Ils ont évalué la réponse à l'anticoagulothérapie initiale en déterminant la recanalisation radiographique des vaisseaux et leur résolution clinique (définie comme l'absence de symptômes continus de thrombose veineuse splanchnique ou de complications exigeant un traitement en plus de l'anticoagulothérapie).

RÉSULTATS : Vingt-deux patients ont participé à l'étude. L'anticoagulothérapie seule a assuré la recanalisation des artères chez $41 \%$ des patients, dont $68 \%$ ont profité d'une résolution clinique. Deux patients ont souffert d'hémorragies. Les facteurs associés à l'absence de résolution clinique incluaient les signes d'hypertension portale ou d'insuffisance hépatique à la présentation, une occlusion complète des vaisseaux au diagnostic, la présence d'un trouble myéloprolifératif ou de la mutation de la tyrosine kinase JAK2 $2^{\mathrm{V} 617 \mathrm{~F}}$ et l'absence de facteur prédisposant local ou transitoire.

CONCLUSIONS : L'anticoagulothérapie semblait efficace comme traitement initial de la thrombose veineuse splanchnique. Des facteurs cliniques peuvent contribuer à dépister les patients vulnérables aux complications et qui ont donc besoin d'une surveillance plus attentive. Ces observations étaient limitées par la petite taille de l'échantillon et devront être évaluées dans le cadre d'études prospectives plus vastes.
Splanchnic vein thrombosis unrelated to primary liver disease is a $\checkmark$ rare and poorly understood clinical phenomenon; information pertaining to its optimal management is limited (1-4). Anticoagulant therapy in patients with splanchnic vein thrombosis has been shown to be effective in recanalizing thrombosed vessels and alleviating symptoms (2). For chronic splanchnic vein thrombosis with ongoing risk factors, prolonged or indefinite anticoagulant therapy has been credited with decreasing recurrence rates and preventing symptom progression $(4,5)$.

When medical management is not effective, progressive or recurrent splanchnic vein thrombosis can cause mesenteric ischemia or portal hypertension requiring surgical or endoscopic management. Given its reported safety and efficacy, most clinicians begin management with anticoagulant therapy alone, resorting to more invasive interventions following an ineffective trial of medical management $(2-4,6)$. In the present study, we aimed to describe our experience with the treatment of splanchnic vein thrombosis and to identify potential predictors of the need for additional interventions beyond anticoagulant therapy.

\section{METHODS}

A retrospective cohort study including consecutive adult patients with a newly diagnosed first episode of noncirrhotic splanchnic vein thrombosis referred to the Thrombosis Clinic at the London Health Sciences Centre in London, Ontario, between January 2008 and August 2011 was conducted. Information regarding demographic and clinical

${ }^{1}$ Department of Surgery, Division of General Surgery, University of Western Ontario; ${ }^{2}$ Multi-Organ Transplant Program, London Health Sciences Centre;

${ }^{3}$ Department of Medicine, Division of Hematology; ${ }^{4}$ Department of Epidemiology and Biostatistics, University of Western Ontario, London, Ontario

Correspondence: Dr Alejandro Lazo-Langner, Division of Hematology, Department of Medicine, University of Western Ontario,

800 Commissioners Road East, Room E6-216A, London, Ontario N6A 5W9. Telephone 519-685-8500 ext 58833, fax 519-685-8477,

e-mail alejandro.lazolangner@lhsc.on.ca

Received for publication November 5, 2014. Accepted February 7, 2014 
TABLE 1

Splanchnic vein involvement and degree of occlusion at maximally occlusive site $(n=22)$

\begin{tabular}{lcccc}
\hline & \multicolumn{3}{c}{ Degree of occlusion, $\mathbf{n}$} & \\
\cline { 2 - 4 } Vessel(s) involved & Complete & Partial & Not specified & Total, $\mathbf{n}(\%)$ \\
\hline Isolated splanchnic vein thrombosis & 2 & 2 & \\
Portal vein & 2 & 5 & 1 & $6(27.3)$ \\
SMV & & & & $6(27.3)$ \\
Multivessel disease & & & 3 & $5(22.7)$ \\
Portal vein + SMV + & 2 & & & \\
$\quad$ SV & & & & $1(4.5)$ \\
Portal vein + SV & 1 & & & $1(4.5)$ \\
Portal vein + SMV & 1 & & & $1(4.5)$ \\
Portal vein + SMV + & 1 & & & $1(4.5)$ \\
$\quad$ DVT & & & & $1(4.5)$ \\
Portal vein + DVT & 1 & & & \\
Portal vein + BCS & 1 & &
\end{tabular}

BCS Budd-Chiari syndrome with hepatic vein thrombosis; DVT Left deep vein thrombosis; SMV Superior mesenteric vein; SV Splenic vein

variables, predisposing factors and thrombophilia was collected. The index date was the date an objectively confirmed diagnosis of portal, splenic and/or superior mesenteric veins was made. Thrombophilia screening was performed in all patients and included prothrombin gene G20210A variant, Factor V Leiden, protein C deficiency, protein S deficiency, antithrombin deficiency, anticardiolipin antibodies, lupus anticoagulant and paroxysmal nocturnal hemoglobinuria. The duration of anticoagulation therapy was also recorded. Patients were classified as having a myeloproliferative disorder if they carried the JAK2 $2^{\mathrm{V} 617 \mathrm{~F}}$ tyrosine kinase mutation (7) or if they met current diagnostic criteria for overt myeloproliferative disorder in the absence of the JAK2 $2^{\mathrm{V} 617 \mathrm{~F}}$ mutation (8).

The response to initial anticoagulant therapy was assessed by determining radiographic recanalization of vessels on follow-up imaging and clinical resolution. The latter was defined as the absence of ongoing splanchnic vein thrombosis symptoms or complications requiring treatment beyond anticoagulant therapy. Also assessed were the occurrence of major bleeding events according to the definition from the International Society on Thrombosis and Haemostasis (9) as well as clinically relevant nonmajor bleeding. Groups were compared using the Mann-Whitney or Fisher's exact tests, as appropriate; $\mathrm{P}<0.05$ was considered to be statistically significant.

\section{Patient characteristics}

\section{RESULTS}

Between January 2008 and August 2011, 22 patients (nine female; mean age 51 years [range 29 to 67 years]) who received anticoagulant therapy and did not demonstrate primary cirrhotic disease were referred to the authors' clinic and included in the study. Table 1 summarizes the distribution of vessels involved. Comorbid conditions included history of deep vein thrombosis or pulmonary embolism $(n=5)$, hypertension $(n=4)$, type 2 diabetes $(n=1)$, obesity $(n=1)$, inflammatory bowel disease $(n=2)$, recent or ongoing nonhepatic malignancy $(n=5)$, and recent abdominal surgery $(n=6)$. A local or transient risk factor for splanchnic vein thrombosis formation was identified in $50 \%$ of the patients. Among the remaining patients, eight had thrombophilic disorders identified (Table 2). Myeloproliferative disorders were identified in six patients $(27.3 \%)$. No underlying risk factors for splanchnic vein thrombosis were identified in three patients.

Four patients were diagnosed incidentally on staging computed tomography scans for colorectal cancer or lymphoma. Another patient was initially imaged due to recurrent, transiently elevated liver enzyme levels. All symptomatic patients were diagnosed with splanchnic vein thrombosis within $24 \mathrm{~h}$ to three weeks from the onset of symptoms.
TABLE 2

Risk factors for splanchnic vein thrombosis $(n=22)$

\begin{tabular}{lc}
\hline Factor & $\mathbf{n ~ ( \% )}$ \\
\hline Local/transient etiology & $11(50)$ \\
Malignancy* & $5(22.7)$ \\
Recent abdominal surgery* $^{*}$ & $7(31.8)$ \\
Idiopathic small bowel obstructions & $1(4.5)$ \\
Diverticulitis & $1(4.5)$ \\
Chronic pancreatitis with recent stenting & $1(4.5)$ \\
Systemic thrombophilia & $8(36.4)$ \\
Myeloproliferative disorder/JAK2V617F tyrosine & $6(27.3)$ \\
kinase mutation ${ }^{\dagger}$ & \\
Prothrombin gene G20210A variant & $2(9.1)$ \\
Factor V Leiden & $4(18.2)$ \\
Multiple thrombophilia & $4(18.2)$ \\
Unknown & $3(13.6)$ \\
\hline
\end{tabular}

*In three patients, abdominal surgery was due to malignancy; + In one patient, myeloproliferative disorder was biopsy proven but JAK2 negative

The most common presenting symptom was abdominal pain (54.5\%). Other presenting complaints included abdominal distension and decreased bowel movements, marked hepatosplenomegaly secondary to myelofibrosis and fever in a patient with a history of recurrent diverticulitis. Of note, one patient presented with upper and lower gastrointestinal bleeding and an acute surgical abdomen secondary to intestinal ischemia. Four patients had documented signs of portal hypertension or hepatic dysfunction at the time of diagnosis of splanchnic vein thrombosis. These included nonbleeding gastroesophageal varices $(n=2)$, hepatosplenomegaly $(n=3)$, ascites $(n=2)$ and jaundice $(n=2)$. Screening endoscopy was not routinely offered to all patients included in the present review, and information pertaining to the presence or absence of gastroesophageal varices was only available for these four patients.

All patients included in the study received anticoagulant therapy promptly after diagnosis. Treatment consisted of unfractionated heparin or low molecular weight heparin followed by oral vitamin $\mathrm{K}$ antagonists. Patients who recurred were switched to long-term treatment with low molecular weight heparin at the discretion of the treating physician who also determined the duration of anticoagulant therapy. All of the 11 patients with only clear local or transient risk factors completed a short course of anticoagulant therapy (six months in 10 patients and three months in a patient whose course was stopped early in anticipation of an unrelated surgical procedure). All remaining patients were prescribed indefinite anticoagulation.

No patients underwent thrombolytic therapy. In one patient who was diagnosed with mesenteric vein thrombosis intraoperatively, visible thrombi were manually removed during laparotomy and anticoagulant therapy was started postoperatively. The mean follow-up period for all patients was 22 months.

Treatment outcomes and predictors

Follow-up imaging was available for 21 patients because one patient died during follow-up from concurrent metastatic colorectal cancer. Vessel recanalization was documented in nine patients (41\%) and clinical resolution was achieved in 15 (68\%). Of the 11 patients who received short-course anticoagulation for splanchnic vein thrombosis associated with only local or transient risk factors, eight $(73 \%)$ obtained vessel recanalization and 10 (91\%) resolved clinically. The local/transient risk factor group had an increased association with recanalization $(\mathrm{P}=0.008)$ and tended to achieve clinical resolution more frequently $(\mathrm{P}=0.063)$ than individuals with identified thrombophilias or unknown etiologies. The clinical complications and subsequent interventions made in patients who did not achieve clinical resolution are descibed in Table 3. 
TABLE 3

Complications and subsequent interventions in patients failing to achieve clinical resolution

\begin{tabular}{|c|c|c|c|}
\hline Patient & Thrombosed vessels & Clinical complications & Interventions \\
\hline 1 & Portal vein & Deceased (ongoing malignancy) & \\
\hline 2 & Portal vein & Development of new nonbleeding varices & Beta-blockers \\
\hline \multirow[t]{3}{*}{3} & Portal vein & Persistence of nonbleeding varices & Beta-blockers \\
\hline & Superior mesenteric vein & Massive splenomegaly + compressive gastric & Splenectomy \\
\hline & Splenic vein & outlet obstruction & \\
\hline \multirow[t]{2}{*}{4} & Portal vein & Transient rethrombosis and abdominal pain & \\
\hline & Splenic vein & & \\
\hline \multirow[t]{3}{*}{5} & Portal vein & Portocaval shunt occlusion & Urgent liver transplant \\
\hline & Hepatic veins (Budd-Chiari syndrome) & Liver failure & \\
\hline & Portocaval shunt & & \\
\hline \multirow[t]{9}{*}{6} & Portal vein & Enlargement of nonbleeding varices & Discontinuation of anticoagulant therapy \\
\hline & Deep vein thrombosis (leg) & & Beta-blockers \\
\hline & & Rethrombosis (deep vein thrombosis) & Resume anticoagulant therapy \\
\hline & & & Splenectomy \\
\hline & & & Devascularization of gastroesophageal junction \\
\hline & & Variceal hemorrhage & Discontinuation of anticoagulant therapy \\
\hline & & & Variceal band ligation \\
\hline & & Rethrombosis: Portal vein & Restart indefinite anticoagulant therapy \\
\hline & & New thrombosis: Inferior vena cava and left renal vein & \\
\hline \multirow[t]{2}{*}{7} & Mesenteric veins (diagnosed intraoperatively) & Acute ischemic gut at initial presentation & Manual thrombectomy \\
\hline & & & Small bowel resection \\
\hline
\end{tabular}

Factors associated with a lack of clinical resolution (and subsequent need for further interventions) included signs of portal hypertension/ liver failure on presentation $(\mathrm{P}=0.005)$, complete vessel occlusion at diagnosis $(\mathrm{P}=0.029)$, presence of a myeloproliferative disorder or JAK2 $2^{\mathrm{V} 617 \mathrm{~F}}$ tyrosine kinase mutation $(\mathrm{P}=0.004)$ and the absence of a local/transient predisposing factor $(\mathrm{P}=0.034)$. There did not appear to be any statistically significant association between outcome and age, sex or clinical presentation. Of the four patients with incidental diagnoses in the context of active or treated malignancy, three attained persistent radiographic recanalization and one died from concurrent metastatic colonic malignancy.

There was one event each of major and clinically relevant nonmajor bleeding. One patient experienced recurrent lower gastrointestinal bleeding that ceased on completion of a six-month course of anticoagulant therapy, and variceal hemorrhage occurred in one patient who subsequently underwent endoscopic variceal band ligation followed by resumption of prolonged anticoagulant therapy for recurrent splanchnic vein thrombosis.

\section{DISCUSSION}

In the present study, we report our experience with the treatment of patients with noncirrhotic splanchnic vein thrombosis using anticoagulant therapy. We found that approximately one-half of the patients achieved recanalization of the involved vessels, particularly among patients with a transient or local risk factor. Other studies have also reported partial or complete recanalization in 33\% to $90 \%$ of cases with anticoagulant therapy alone $(1,2,4,10)$. However, in addition to vessel recanalization, the presence of clinical resolution (and avoidance of additional therapies) is also a clinically relevant parameter that has not been frequently studied. In fact, studies investigating lower-extremity deep vein thrombosis have not demonstrated that radiographic resolution alone is associated with improved rates of symptom-free survival or recurrence (11). In this regard, we found that clinical resolution occurred more frequently in patients with transient/ local risk factors, in those with partial vein occlusions, in those without signs of portal hypertension or liver failure, and in those without the JAK2 $2^{\mathrm{V} 617 \mathrm{~F}}$ mutation. This information may help to identify patients at higher risk for developing chronic complications and, thus, requiring closer monitoring. There did not appear to be any statistically significant association between outcome and age, sex or clinical presentation in the current study.

In patients with ongoing prothrombotic disorders, prolonged anticoagulant therapy has been found to decrease the rate of recurrent thrombotic complications by $>60 \%(4,5)$. While there are no trial data available to identify specific patient groups in which prolonged anticoagulant therapy is necessary to prevent splanchnic vein thrombosis complications, some authors suggest prescribing long-term anticoagulant therapy for all patients with thrombophilic disorders and any patient who presents with mesenteric vein thrombosis due to the risk of recurrent bowel ischemia $(1,3,5)$.

However, the use of anticoagulant therapy has been associated with an increased risk for bleeding, particularly in the presence of additional risk factors. Therefore, the risk of using anticoagulant therapy should be carefully considered in each patient. Based on a retrospective cohort study involving 136 patients, Condat et al (5) reported no significant association between anticoagulant therapy and the risk or severity of variceal bleeding in splanchnic vein thrombosis patients. They published a risk-benefit ratio that favoured the use of anticoagulant therapy to prevent thrombus progression, thereby preventing further increases in portal pressure (5). In contrast, some authors have advised against the use of long-term anticoagulant therapy in light of significant variceal hemorrhage rates; however, these recommendations were based on study populations that included patients with cirrhotic splanchnic vein thrombosis (12). The natural history of variceal bleeding in primary splanchnic vein thrombosis patients appears to differ from that of cirrhotic patients, with decreased severity when matched for similar variceal characteristics, decreased frequency of bleeds and overall better outcomes $(3,6)$. This may be due to an intact coagulation pathway in the context of normal liver function (3). Even in cirrhotic patients, however, anticoagulation may be beneficial. In a recent randomized trial involving 70 advanced cirrhotic patients, Villa et al (13) demonstrated that prophylactic low molecular weight heparin was both safe and effective in preventing the development of portal vein thrombosis. They cited improved survival in the anticoagulation group, less hepatic decompensation and no bleeding complications.

In cirrhotic and noncirrhotic patients, variceal size, endoscopic features and a history of previous bleeds can help to predict future 
bleeding risk and may guide the decision to proceed with endoscopic treatment before anticoagulant therapy or to institute a thorough endoscopic screening protocol. It may be advisable to screen all patients with portal venous thrombosis endoscopically (14). Alternatively, one could restrict endoscopic assessment to patients with ultrasonographic features suggestive of esophageal varices (15). While some have proposed that the presence of nonbleeding varices may be an indication to hold or shorten anticoagulation courses (12), this does not optimally minimize morbidity and mortality due to the poor outcomes of progressive splanchnic vein thrombosis. Recanalization is the best treatment for varices formed secondary to splanchnic thrombosis and, as such, withholding anticoagulation should probably be discouraged in the absence of high bleeding risk (16). Beta blockade remains an effective means of preventing variceal bleeding in general (3), although variceal band ligation may be preferable in the setting of portal vein thrombosis (14) and is effective $90 \%$ of the time in hemorrhage prevention (16). A dual approach of endoscopic and medical management has led to more pronounced variceal regression (14). Therefore, a pragmatic approach may involve prophylactic endoscopic variceal band ligation with concurrent anticoagulant therapy (3). In the setting of variceal hemorrhage, endoscopic eradication significantly reduces the risk of recurrent bleeding in these patients, allowing for ongoing anticoagulation for treatment of their underlying thrombotic pathology (3).

Taking the previous information into consideration, it is our practice to consider anticoagulation for all patients with newly diagnosed splanchnic vein thrombosis if there is no contraindication, particularly esophageal varices with endoscopic high-risk bleeding features. For patients with events associated with transient risk factors, we consider three to six months of anticoagulation. For patients without associated risk factors and in the presence of high-risk thrombophilias (eg, combined defects, antiphospholipid antibodies, myeloproliferative neoplasms), we consider indefinite anticoagulation. However, periodic reassessments are performed to re-evaluate bleeding risk and thrombus resolution. In patients without evidence of improvement during followup (eg, thrombus persistence with development of collaterals and/or cavernomatous transformation), we consider discontinuation of anticoagulants due to futility.

Given the efficacy and safety of anticoagulant therapy and the overall low mortality rate associated with splanchnic vein thrombosis, the general consensus is to reserve invasive procedures such as transjugular intrahepatic portosystemic shunt procedures for cases in which anticoagulant therapy and endoscopy are ineffective $(2,3,6,10)$. Additionally, several surgical techniques have been used in this patient population, including splanchnic-intrahepatic portal bypass, splenectomy and devascularization of the gastroesophageal junction, which has reportedly been more effective in spontaneous splanchnic vein thrombosis than in cirrhotic patients (3). Given the heterogeneity of splanchnic vein thrombosis, its complications and its predisposing factors, the choice of interventions is difficult to standardize and is best informed by patient factors.

In addition to splanchnic vein thrombosis itself, concomitant diseases - particularly cancer and myeloproliferative disorders - are an important cause of morbidity and mortality in these patients $(3,4,6)$. The relatively recent description of the association between splanchnic vein thrombosis and the presence of the $J A K 2^{\mathrm{V} 617 \mathrm{~F}}$ tyrosine kinase mutation found in myeloproliferative disorders should prompt its investigation in all patients because these disorders require further hematological work-up $(7,17,18)$. In addition, some authors suggest screening for thrombophilic disorders in splanchnic vein thrombosis patients regardless of apparent local etiology because both local and thrombophilic factors are believed to interact in the pathogenesis of splanchnic vein thrombosis $(4,19)$. It is our practice to investigate patients with idiopathic noncirrhotic splanchnic vein thrombosis for thrombophilia, although the clinical value of this practice is yet to be determined. Finally, the role of screening for other potential prothrombotic conditions, such as paroxysmal nocturnal hemoglobinuria, is at present unclear, particularly given its rarity, although patients with this condition have been reported to frequently develop splanchnic vein thrombosis (20).

The limitations of the present study were those inherent in its single-centre retrospective design, as well as the observational approach required to analyze a rare, heterogenous and potentially lifethreatening condition. Another limitation was that six of our patients had the diagnosis made incidentally on computed tomography scan. The significance of an incidental finding of splanchnic vein thrombosis is not certain. Finally, other limitations include the small study population and nonstandardized treatment and screening algorithms. In the absence of randomized controlled trials, observational studies may help to inform treatment practices; however, our findings should certainly be regarded as hypothesis generating.

\section{CONCLUSION}

Based on our experience and that of others, the use of initial anticoagulant therapy appears to be a reasonable option in the initial management of patients with splanchnic vein thrombosis in the absence of contraindications to anticoagulation. The presence of certain clinical factors may help to identify patients at higher risk for developing chronic complications and need for further therapy, thus requiring closer monitoring. These findings need to be further explored in larger prospective studies to provide robust clinical recommendations.

DISCLOSURES: Presented in poster format at the Canadian Surgery Forum, Calgary Alberta, in September 2012 and at the American Society of Hematology Annual Meeting, Atlanta, Georgia, USA, in December 2012.

\section{REFERENCES}

1. Amitrano L, Guardascione MA, Scaglione M, et al. Prognostic factors in noncirrhotic patients with splanchnic vein thromboses. Am J Gastroenterol 2007;102:2464-70.

2. Condat B, Pessione F, Denninger MH, et al. Recent portal or mesenteric vein thrombosis: Increased recognition and frequent recanalization on anticoagulant therapy. Hepatology 2000;32:465-70.

3. Webster GJM, Burroughs AK, Riordan SM. Review article: Portal vein thrombosis - new insights into aetiology and management. Aliment Pharmacol Ther 2005;21:1-9.

4. Turnes J, Garcia-Pagan JC, Gonzalez M, et al. Portal hypertensionrelated complications after acute portal vein thrombosis: Impact of early anticoagulation. Clin Gastroenerol Hepatol 2008;6:1412-7.

5. Condat B, Pessione F, Hillaire S, et al. Current outcome of portal vein thrombosis in adults: Risk and benefit of anticoagulant therapy. Gastroenterology 2001;120:490-7.

6. Janssen HLA, Wijngoud A, Haagsma EB, et al. Extrahepatic portal vein thrombosis: Aetiology and determinants of survival. Gut 2001;49:720-4.

7. Orr DW, Patel RK, Lea NC, et al. The prevalence of the activating JAK2 tyrosine kinase mutation in chronic porto-splenomesenteric venous thrombosis. Alimen Pharmacol Ther 2010;31:1330-6.

8. Tefferi A, Vardiman JW. Classification and diagnosis of myeloproliferative neoplasms: The 2008 World Health Organization criteria and point-of-care diagnostic algorithms. Leukemia 2008;22:14-22.

9. Schulman S, Kearon C; Subcommittee on Control of Anticoagulation of the Scientific and Standardization Committee of the International Society on Thrombosis and Haemostasis. Definition of major bleeding in clinical investigations of antihemostatic medicinal products in non-surgical patients. J Thromb Haemost 2005;3:692-4.

10. Plessier A, Darwish-Murad S, Hernandez-Guerra M, et al. Acute portal vein thrombosis unrelated to cirrhosis: A prospective multi-centre follow-up study. Hepatology 2010;51:210-8.

11. Rodger MA, Kahn SR, Wells PS, et al. Identifying unprovoked thromboembolism patients at low risk for recurrence who can discontinue anticoagulant therapy. CMAJ 2008;179:417-26.

12. Thatipelli MR, McBane RD, Hodge DO, et al. Survival and recurrence in patients with splanchnic vein thrombosis. Clin Gastroenterol Hepatol 2010;8:200-5. 
13. Villa E, Camma C, Marietta M, et al. Enoxaparin prevents portal vein thrombosis and liver decompensation in patients with advanced cirrhosis. Gastroenterology 2012;143:1253-60.

14. Sogaard KK, Astrup LB, Vilstrup H, et al. Portal vein thrombosis; risk factors, clinical presentation and treatment. BMC Gastroenterol 2007;7:34.

15. Brintintan A, Chira RI, Mircea PA. Non-invasive ultrasound-based diagnosis and staging of esophageal varices in liver cirrhosis. A systematic review of the literature published in the third millennium. Med Ultrason 2013;15:116-24.

16. Spahr L, Boehlen F, de Moerloose P, et al. Anticoagulants in portal vein thrombosis: Don't be so shy! Blood 2009;113:5031-2.

17. Regina S, Herault O, D'Alteroche L, et al. JAK2 V617F is specifically associated with idiopathic splanchnic vein thrombosis. J Thromb Haemost 2007;5:859-61.
18. De Stefano V, Rossi E, Za T, et al. JAK2 V617F mutational frequency in essential thrombocythemia associated with splanchnic or cerebral vein thrombosis. Am J Hematol 2011;86:526-8.

19. Denninger MH, Chait Y, Casadevall N, et al. Cause of portal or hepatic venous thrombosis in adults: The role of multiple concurrent factors. Hepatology 2000;31:587-91.

20. Lee JW, Jang JH, Kim HS, et al. Clinical signs and symptoms associated with increased risk of thrombosis in patients with paroxysmal nocturnal hemoglobinuria from a Korean registry. Int J Hematol 2013;97:749-57. 


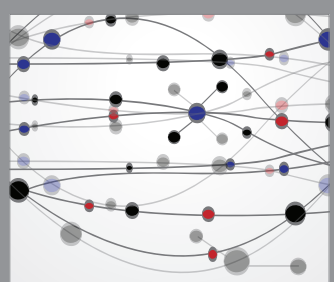

The Scientific World Journal
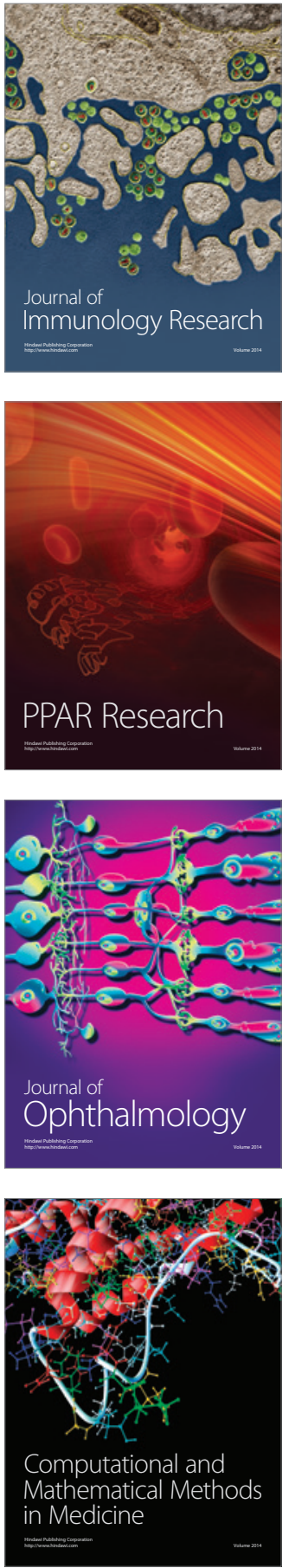

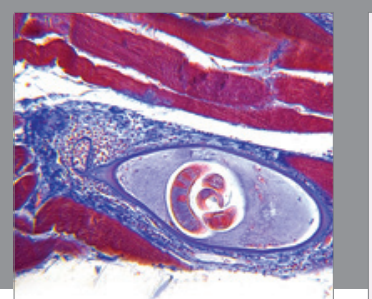

Gastroenterology Research and Practice

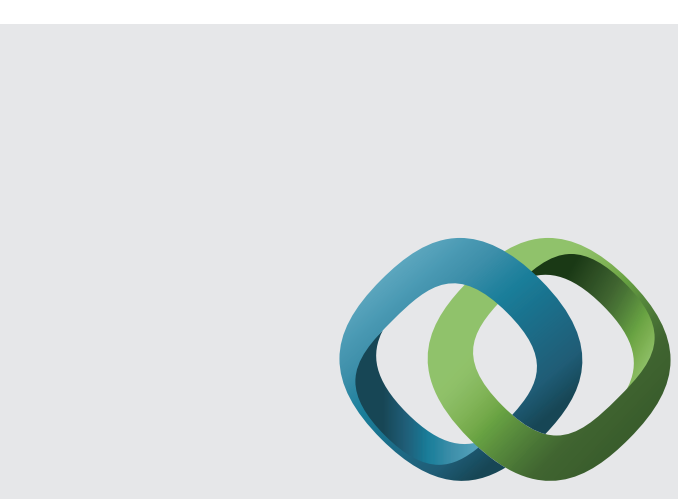

\section{Hindawi}

Submit your manuscripts at

http://www.hindawi.com
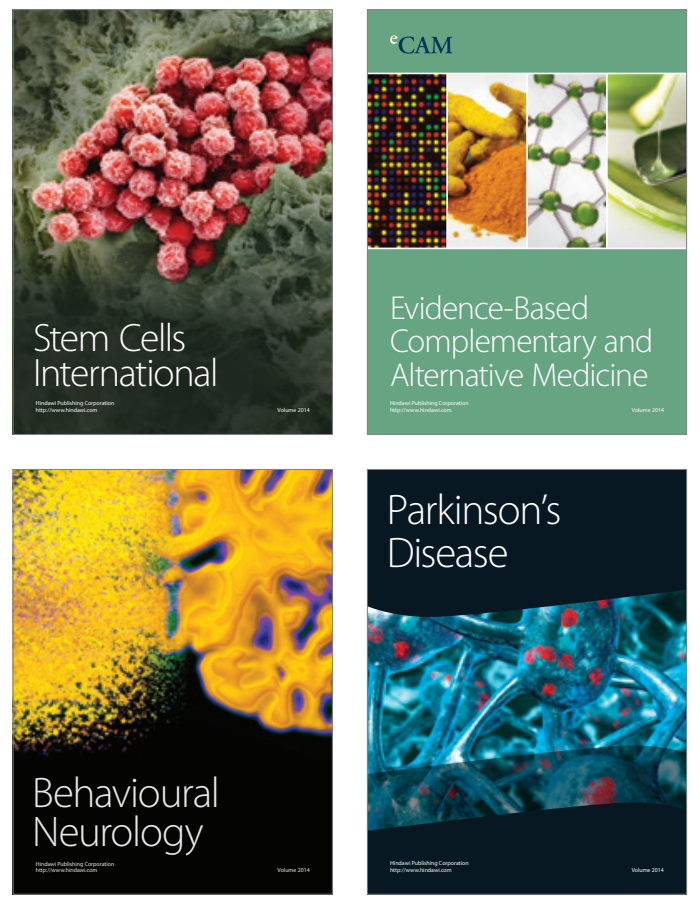
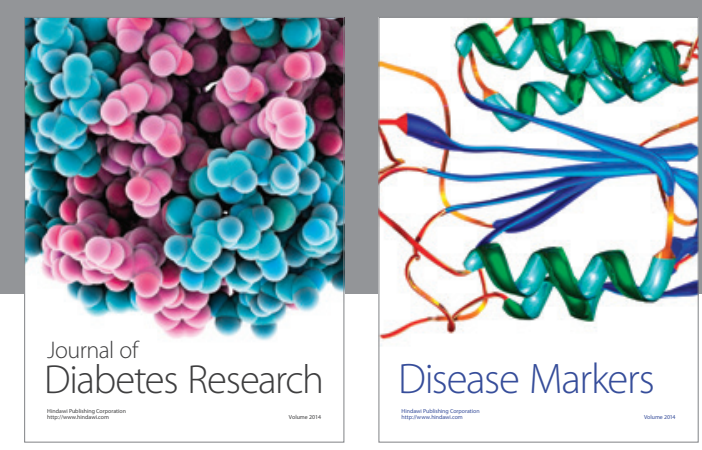

Disease Markers
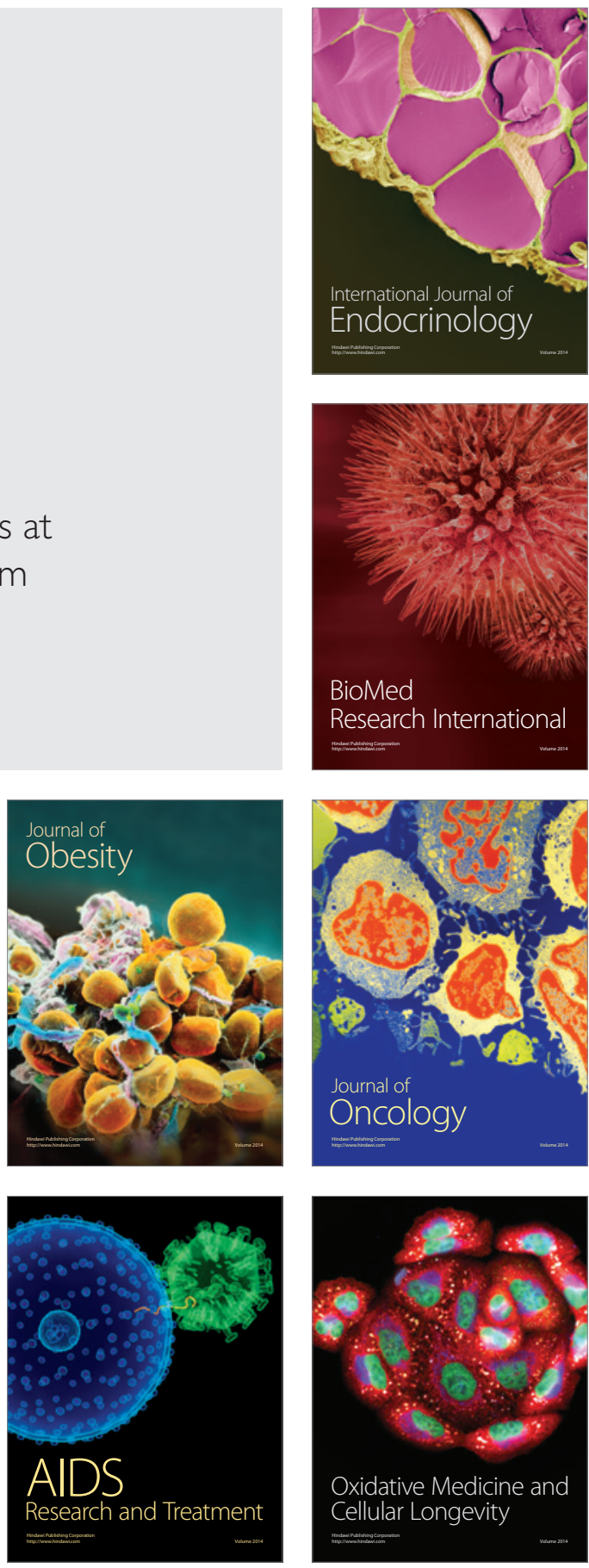